Article

\title{
What Is the Impact of Microplastics and Lipid Regulators on Marine Meiofauna? Case Study of Polyvinyl Chloride, Atorvastatin, and Simvastatin
}

\author{
Mohamed Allouche ${ }^{1,+}$, Sahar Ishak ${ }^{1,+} \mathbb{D}$, Ahmed Nasri ${ }^{1}$, Amor Hedfi ${ }^{2} \mathbb{D}$, Manel Ben Ali ${ }^{2}$, Bander Albogami ${ }^{2} \mathbb{D}$, \\ Mohammed Almalki ${ }^{2}$, Octavian Pacioglu ${ }^{3}$ and Fehmi Boufahja ${ }^{1, *}$
}

1 LR01ES14 Laboratory of Environment Biomonitoring, Coastal Ecology and Ecotoxicology Unit, Faculty of Sciences of Bizerte, University of Carthage, Zarzouna 7021, Tunisia; allouchemom2@gmail.com (M.A.); ishak-sahar@hotmail.com (S.I.); a7mednas@gmail.com (A.N.)

2 Department of Biology, College of Sciences, Taif University, P.O. Box 11099, Taif 21944, Saudi Arabia; o.zaied@tu.edu.sa (A.H.); mjbinali@tu.edu.sa (M.B.A.); b.boqami@tu.edu.sa (B.A.); almalki.m@tu.edu.sa (M.A.)

3 National Institute of Research and Development for Biological Sciences, 060031 Bucharest, Romania; octavian.pacioglu@e-uvt.ro

* Correspondence: fehmiboufahja@yahoo.fr

+ These authors contributed equally to this publication.

\section{check for} updates

Citation: Allouche, M.; Ishak, S.; Nasri, A.; Hedfi, A.; Ben Ali, M.; Albogami, B.; Almalki, M.; Pacioglu, O.; Boufahja, F. What Is the Impact of Microplastics and Lipid Regulators on Marine Meiofauna? Case Study of Polyvinyl Chloride, Atorvastatin, and Simvastatin. Sustainability 2021, 13, 13190. https://doi.org/10.3390/ su132313190

Academic Editor: Alejandro Rescia

Received: 30 October 2021

Accepted: 18 November 2021

Published: 29 November 2021

Publisher's Note: MDPI stays neutral with regard to jurisdictional claims in published maps and institutional affiliations.

Copyright: (c) 2021 by the authors. Licensee MDPI, Basel, Switzerland. This article is an open access article distributed under the terms and conditions of the Creative Commons Attribution (CC BY) license (https:/ / creativecommons.org/licenses/by/ $4.0 /)$.
Abstract: A microcosm experiment was carried out to provide a deeper insight into the toxic mechanisms exerted by two lipid regulator agents, as well as their interactions with the polyvinyl chloride microplastic on marine meiofauna. Two concentrations of Atorvastatin " $A$ " and of Simvastatin " $S$ ", (i.e., $0.6 \mathrm{mg} . \mathrm{kg}^{-1}$ and $6 \mathrm{mg} \cdot \mathrm{kg}^{-1}$ ), as well as a single dosage of polyvinyl chloride microplastics " $\mathrm{P}$ " at $20 \mathrm{mg} \cdot \mathrm{kg}^{-1}$, separately and their combined mixtures (" $\mathrm{AP}$ " and "SP") were used on coastline dwelling marine meiofauna, with a main focus on nematodes. The results showed a significant reduction in meiofauna abundance in treatments compared to control. SIMPER analysis highlighted a significant decrease in the abundance of epigrowth feeders (2A), which possess conical (co) tails, and indistinct (id) amphideal foveas compared to control microcosms, reflected mainly in the decrease in abundance of the species Prochromadorella longicaudata. Furthermore, the contamination with microplastic affected only the omnivores-carnivores guild. Another finding of the current experiment is that the mixtures of microplastic with drugs lead to synergic interactions that increased their toxic effects on marine nematode communities.

Keywords: lipid regulators; polyvinyl chloride (PVC); meiobenthic nematodes; taxonomy; functional traits

\section{Introduction}

Seafood adequate quality and safety is a major challenge in food sciences and in most fisheries and aquaculture research departments [1]. The ecotoxicological effects of pollutants in marine habitats represent a topic of growing concern. An important class of such chemicals comprises the over-the-counter and prescription drugs by humans at an alarmingly increasing rate. It was reported that up to $90 \%$ of these drugs end in aquatic habitats via human egesta through wastewater, leaks from septic tanks, and landfills [2].

The increasing consumption of cardiovascular drugs and lipid regulator is currently recorded at alarming rates in western countries, such as Germany, UK, and Canada [3-5]. In the USA, the most widespread lipid regulator agents are Simvastatin "S" (hereafter S) and Atorvastatin "A" (hereafter A) [6,7]. Statins comprise lipid regulators that inhibit the cholesterogenesis in liver, hence reducing the cholesterol production in the human body [8]. These compounds are known to induce toxic effects on aquatic invertebrates too, as it was proved for A [9] and S [10], respectively. Moreover, these compounds are absorbed by other organic compounds in marine sediments [11,12]. 
Another major class of emerging pollutants in marine habitats are the microplastics. Whereas plastic particles $>5 \mathrm{~mm}$ in diameter can be consumed by marine biota [13], smaller particles, defined as microplastics [14,15], are not easily removable from the marine environment [16]. The microplastics represent nowadays one of the most undesirable and widespread types of contaminants in marine habitats [17-20]. Of special concern is the polyvinyl chloride (hereafter $\mathrm{P}$ ), which adsorbs a variety of other pollutants, such as drugs and heavy metals [21-24].

Free-living marine nematodes comprise a very diverse and abundant group of benthic organisms [25]. These worms represent a key-group essential for the wellbeing of marine benthic habitats; hence their wide usage as bioindicators in routine environmental biomonitoring programs [26-30]. Their small body-size and fast life cycle, associated with the ease of their maintenance in laboratory conditions, makes this group very useful in ecotoxicological studies [31-33]. Our aim was to investigate the multifaceted effects of single (i.e., A, S and P, respectively), as well as of their combined (i.e., AP and SP) interactions on the abundance, diversity, and potential changes in the ratio of functional traits of typical coastline dwelling nematode communities. We expected polyvinyl chloride and lipid regulators to induce visible negative effects on all meiobenthic taxa, as well as for their mixture. Another working hypothesis is that the effect would be of additive nature given no chemical interactions are known among the tested xenobiotics.

\section{Material and Methods}

\subsection{Collecting Site and Sediment Manipulation}

Sediments were collected on 1 December 2020 from a subtidal site $\left(37^{\circ} 16^{\prime} 435^{\prime \prime} \mathrm{N}\right.$, $9^{\circ} 52^{\prime} 453^{\prime \prime}$ E) within the harbor of Bizerte, Tunisia. Multiple hand cores $\left(10 \mathrm{~cm}^{2}\right.$ area $)$ were employed to sample the top layer (i.e., $5 \mathrm{~cm}$ ) of sediment at $50 \mathrm{~cm}$ below water surface, according to Hedfi [34]. The sampling was restricted to the first $5 \mathrm{~cm}$ of sediment because, in most coastal habitats with fine sediments, more than $90 \%$ of the meiofauna are found in the surface 1-2 cm [34]. The collected sediment was stored in a room with fixed lighting $(10.5 \mathrm{~h}$ light $/ 13.5 \mathrm{~h}$ dark $)$ and temperature $\left(2{ }^{\circ} \mathrm{C} / 16{ }^{\circ} \mathrm{C}\right)$ for three days of acclimatization. The room temperature used in the current experiment was based on the temperature recorded (http:/ / www.infoclimat.fr accessed on 30 November 2020) for the previous month (i.e., November 2020).

\subsection{Sediment Contamination and Experiment Set-Up}

Before the experiment, a part of the collected sediments was defaunated by repeated freezing ( 3 times) at $-20^{\circ} \mathrm{C}$ and thawing $(12 \mathrm{~h} / 48 \mathrm{~h})$, according to Schratzberger et al. [35]. Following the removal of coarser particles by sieving $(1 \mathrm{~mm})$, the rest of the sediment was kept for acclimatization for one week. Simultaneously, a stock solution $\left(2 \mathrm{~g} . \mathrm{L}^{-1}\right)$ of A and $\mathrm{S}$ (Sigma-Aldrich) was diluted in dimethyl sulfoxide (DMSO) and kept in dark at room temperature. The stock solution was used to enrich $100 \mathrm{~g}$ of defaunated sediment for sediment concentrations of $0.6 \mathrm{mg} \cdot \mathrm{kg}^{-1}$ of A1 and S1 and $6 \mathrm{mg} \cdot \mathrm{kg}^{-1}$ of A2 and S2, respectively. The sediment concentrations of A and S were chosen based on invertebrates LC 50/96 h used in toxicological studies [9,10]. The experiment lasted for one month [31-33]. Spherical particles of microplastics (>40 $\mu \mathrm{m}$; MW $=48,000 \mathrm{~g} \cdot \mathrm{mol}^{-1}, 16 \mathrm{mmol}$ of $\mathrm{Cl} . \mathrm{g}^{-1}, 99 \%$ ) were added as such as to contaminate $100 \mathrm{~g}$ of defaunated sediment with a concentration of $20 \mathrm{mg} \cdot \mathrm{kg}^{-1}[36]$.

Overall, 30 sets were prepared (three replicates for each set) using a factorial design approach: one set comprised the control, one set of sediment was contaminated with $\mathrm{P}$, two sets of sediments were contaminated with the employed concentrations of $\mathrm{A}$, and the other two with S. Moreover, two sets were contaminated with a mixture of $\mathrm{P}$ and each of the A concentrations; two sets of sediments contaminated by a mixture of $\mathrm{P}$ and each of the $\mathrm{S}$ concentrations.

Each microcosm was filled with $200 \mathrm{~g}$ of sediment with natural meiofauna mixed with $100 \mathrm{~g}$ of contaminated defaunated sediment and topped with one liter of filtered water 
$(40 \mu \mathrm{m})$. The control experimental set " $\mathrm{C}$ " was obtained by mixing $100 \mathrm{~g}$ uncontaminated defaunated sediment with $200 \mathrm{~g}$ of natural sediment. Each system consisted of a 2-litre glass bottle, which was constantly aerated through an aquarium pump throughout the experiment $[34,36]$.

\subsection{Meiofauna Analyses}

The meiofauna was removed from the sediment using two sieves with mesh sizes of $1 \mathrm{~mm}$ and $40 \mu \mathrm{m}$, respectively [37], then stained with Rose-Bengal $\left(0.2\right.$ g.L $\left.{ }^{-1}\right)$ [38] and fixed in $4 \%$ formalin [35]. Afterwards, 100 randomly collected nematodes per replicate were removed under a dissecting microscope and transferred into $21 \%$ glycerol vials [39]. Species identification was done using the taxonomic keys of Platt and Warwick [40,41], Warwick et al. [42], as well as the Ghent University database [43]. Five functional traits were also quantified in the current experiment:

Feeding groups classified according to Wieser [44] as follows: selective deposit feeders (1 A), epigrowth feeders (2 A), and omnivores/predators (2 B), non-selective deposit feeders (1 B).

- The shape of tails, according to Thistle et al. [45] as follows: conical (co), short/round (s/r), clavate/conico-cylindrical (cla), and elongated/filiform (e/f).

- The shape of amphideal fovea, according to Semprucci et al. [29], as follows: spiral (Sp), circular (Cr), indistinct (Id), and pocket (Pk).

- The life history (c-p scores), according to Bongers et al. [46] ranked from 1 to 5.

- The length of adults, according to Schratzberger et al. [47] divided in the following size-classes: 1-2 mm, 2-4 mm, and $>4 \mathrm{~mm}$. The body-size was measured using a Nikon DS-Fi2 camera coupled with a Nikon microscope (Image Software NIS Elements Analysis Version 4.0 Nikon 4.00.07ebuild 787e64 bit).

\subsection{StatisticalAanalyses}

The abundance of nematodes $(\mathrm{N})$, taxonomic richness $(\mathrm{S})$, the Shannon-Wiener index $\left(\mathrm{H}^{\prime}\right)$, and Pielou's evenness $\left(\mathrm{J}^{\prime}\right)$ were calculated using PRIMER v5.0 software [48,49], after data normalization (Kolmogorov-Smirnov test) and assessing the homogeneity of variance (Bartlett test). A $\log _{10}(x+1)$ transformation was applied to data for parametric analyses $[48,50]$. One-way ANOVA, followed by multiple post hoc comparison Tukey HSD tests were performed in STATISTICA (v5.1) to check the differences of the measured metrics among treatments. Multidimensional non-metric ordination (nMDS) was performed using species abundance data, following their square roots transformation, and was based on Bray-Curtis similarity matrix. ANOSIM analysis was used to determine possible significant differences between the nematode assemblages from different types of microcosms. SIMPER analysis (similarity percentage) allowed the identification of most contributing species to the dissimilarity/similarity among control and treatments. Another nMDS ordination was performed to identify which functional traits influenced the response of various species to treatments.

\section{Results}

\subsection{Meiofaunistic Abundances}

The control microcosms were dominated by the end of the experiment by free-living marine nematodes (1154.66 \pm 79.54 individuals/microcosm) (ind.mic $\left.{ }^{-1}\right)$. The second most abundant group was the copepods (108 \pm 16.64 ind.mic $\left.{ }^{-1}\right)$, followed by amphipods $\left(2.66 \pm 0.57\right.$ ind. $\left.\mathrm{mic}^{-1}\right)$, and polychaetes $\left(2.33 \pm 0.57\right.$ ind. $\left.\mathrm{mic}^{-1}\right)$ (Figure 1). 


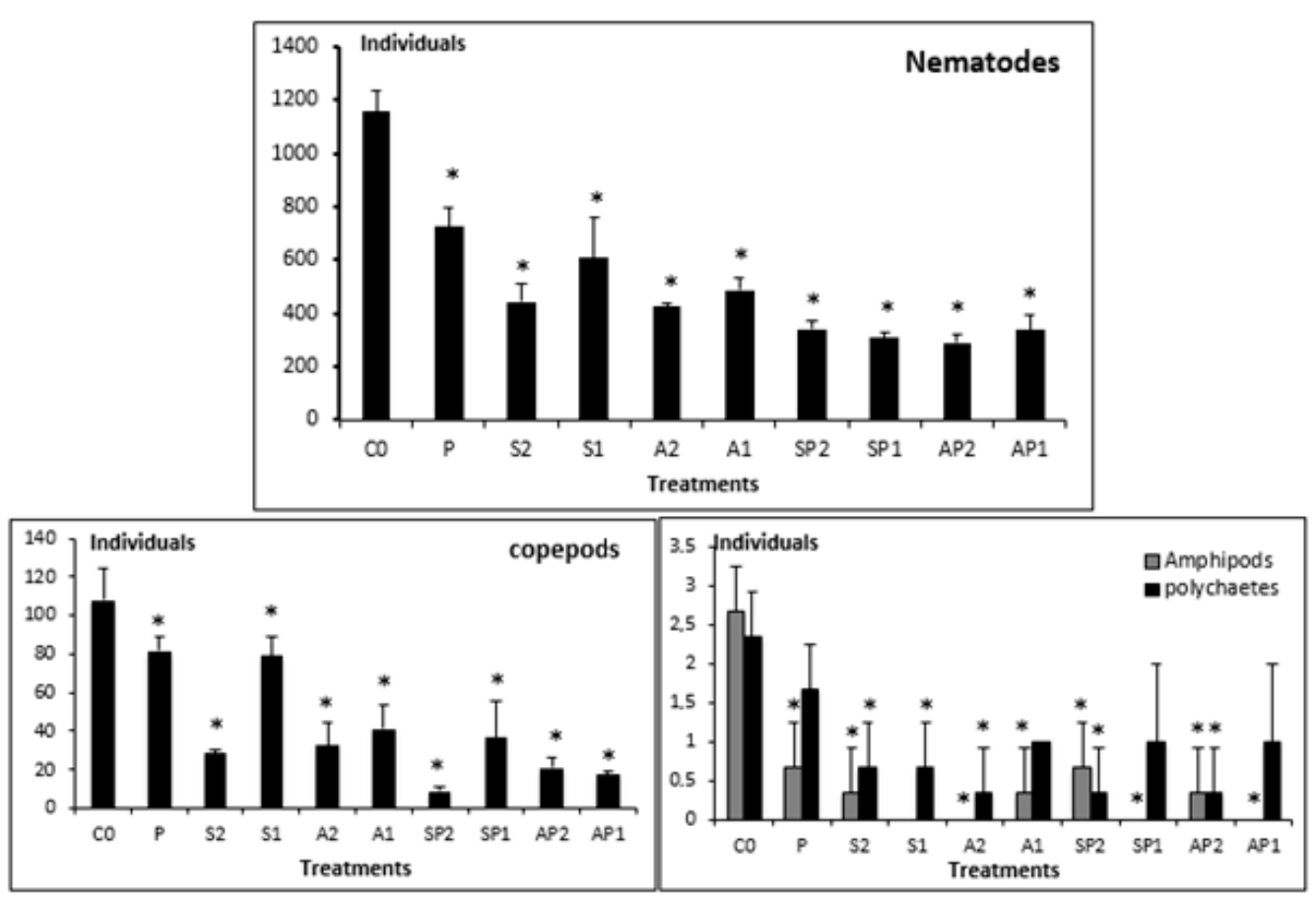

Figure 1. Abundances of meiobenthic taxa from uncontaminated (C0) and contaminated (P; S2; S1; A2; A1; SP2; SP1; AP2; AP1) microcosms. $\left({ }^{*}\right)$ above bars indicate significant difference $(p<0.05$.)

Compared to control experimental sets, a significant reduction $(p<0.05$, Tukey's HSD test, Figure 1) in nematodes' abundance was observed, mainly in treatment AP2 $\left(290.33 \pm 31.34\right.$ ind. $\left.\mathrm{mic}^{-1}\right)$, which showed the lowest abundance. Likewise, the copepod abundance showed a significant decrease in all treatments, mainly in SP2 $\left(9 \pm 2\right.$ ind.mic $\left.{ }^{-1}\right)$. The abundance of polychaetes and amphipods followed a similar pattern (Figure 1).

\subsection{Taxonomic Diversity of Nematodes}

The free-living marine nematodes comprised 20 species spanning 5 orders, 12 families, and 18 genera; the families Xyalidae and Oncholaimidae were dominant (Table 1).

By the end of the experiment the control treatment (C0) was dominated by Prochromadorella longicaudata $(30.67 \pm 1.53 \%)$, Oncholaimus campylocercoides $(16.67 \pm 1.15 \%)$, Anticoma eberthi $(6 \pm 1 \%)$, and Metalinhomoeus numidicus ( $6 \pm 1 \%)$, respectively, comprising less than $5 \%$ of the nematofauna after one month-exposure. In treatments $\mathrm{P}$, a similar trend was observed for the diversity indices, the community being dominated by $P$. longicaudata $(28.33 \pm 1.53 \%)$. The treatments S2 $(17.67 \pm 3.21 \%)$, S1 $(18.67 \pm 1.53 \%)$, A2 $(16.67 \pm 0.58 \%)$, and A1 $(21 \pm 1 \%)$ were dominated by O. campylocercoides. The same species dominated the treatments SP2 $(15.33 \pm 0.58 \%), \mathrm{SP} 1(16.33 \pm 1.53 \%)$, AP2 $(16.67 \pm 0.58 \%)$ and "AP1" $(21 \pm 1 \%)$ (Table 1$)$.

The species richness $(\mathrm{S})$ and diversity indices $\left(\mathrm{d}\right.$ and $\left.\mathrm{H}^{\prime}\right)$ revealed significant differences compared to C0 ( $p<0.05$, Tukey's HSD test, Figure 2$)$, revealing a surprisingly increase of the overall nematode diversity (Figure 2).

The nMDS output indicated a significant effect induced by S, A, and P alone or by their mixtures on species distribution within the ordination space (stress $=0.2$ ). The AP2 treatment was most dissimilar compared to $\mathrm{C} 0$, whereas the $\mathrm{P}$ community was the closest to control (Figure 3). 


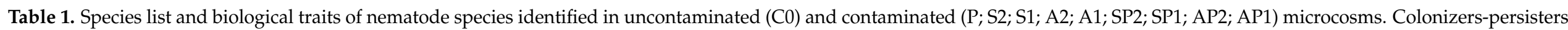

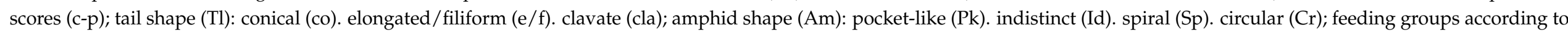
Wieser (1953) (FG): selective deposit-feeders (1A). non-selective deposit-feeders (1B). epistratum-feeders (2A). omnivores carnivores (2B); adult length (AL).

\begin{tabular}{|c|c|c|c|c|c|c|c|c|c|c|c|c|c|c|c|}
\hline \multirow[t]{2}{*}{ Species } & \multicolumn{5}{|c|}{ Functional Traits } & \multicolumn{10}{|c|}{ Treatments } \\
\hline & c-p & $\mathrm{Tl}$ & Am & FG & $\begin{array}{l}\text { Adult } \\
\text { Length }\end{array}$ & $\mathrm{CO}$ & $\mathbf{P}$ & S2 & S1 & A2 & A1 & SP2 & SP1 & AP2 & AP1 \\
\hline $\begin{array}{l}\text { Anticoma } \\
\text { acuminata }\end{array}$ & 2 & $e / f$ & $\mathrm{pk}$ & $1 \mathrm{~A}$ & $2-4 \mathrm{~mm}$ & $0.91 \pm 0.78$ & $0.46 \pm 0.8$ & & $0.43 \pm 0.75$ & $0.47 \pm 0.81$ & $0.42 \pm 0.72$ & $1.63 \pm 0.7$ & $0.96 \pm 0.83$ & $0.44 \pm 0.76$ & $1.25 \pm 1.27$ \\
\hline $\begin{array}{l}\text { Anticoma } \\
\text { eberthi }\end{array}$ & 2 & $e / f$ & pk & $1 \mathrm{~A}$ & $2-4 \mathrm{~mm}$ & $8.27 \pm 1.15$ & $13.01 \pm 1.37$ & $13.55 \pm 1.85$ & $10.9 \pm 1.85$ & $5.35 \pm 1.09$ & $14.75 \pm 1.06$ & $14.28 \pm 1.78$ & $6.74 \pm 0.88$ & $8.32 \pm 1.93$ & $14.29 \pm 3.02$ \\
\hline $\begin{array}{l}\text { Calomicrolaimus } \\
\text { honestus }\end{array}$ & 3 & co & $\mathrm{sp}$ & $2 \mathrm{~A}$ & $1-2 \mathrm{~mm}$ & $2.75 \pm 1.34$ & $1.38 \pm 1.37$ & $1.26 \pm 1.24$ & $3.94 \pm 2.3$ & $1.8 \pm 0.79$ & $2.06 \pm 1.46$ & $1.63 \pm 0.7$ & $3.37 \pm 0.85$ & $1.75 \pm 0.76$ & $1.2 \pm 1.19$ \\
\hline $\begin{array}{c}\text { Chromadora } \\
\text { sp. }\end{array}$ & 2 & co & id & $2 \mathrm{~A}$ & $1-2 \mathrm{~mm}$ & $1.83 \pm 0.75$ & $4.19 \pm 0.09$ & $0.86 \pm 0.75$ & $0.84 \pm 1.48$ & $0.45 \pm 0.78$ & & $1.22 \pm 1.22$ & $0.97 \pm 0.84$ & $0.43 \pm 0.75$ & \\
\hline $\begin{array}{l}\text { Daptonema } \\
\text { trabeculosum }\end{array}$ & 2 & cla & $\mathrm{cr}$ & $1 \mathrm{~B}$ & $1-2 \mathrm{~mm}$ & $1.38 \pm 1.35$ & $2.79 \pm 0.06$ & $5.08 \pm 1.26$ & $1.75 \pm 1.98$ & $8.13 \pm 1.72$ & $4.92 \pm 1.22$ & $2.44 \pm 1.21$ & $3.85 \pm 1.69$ & $8.79 \pm 2.12$ & $6.1 \pm 1.18$ \\
\hline $\begin{array}{c}\text { Marylynnia } \\
\text { puncticaudata }\end{array}$ & 3 & $e / f$ & $\mathrm{sp}$ & $2 \mathrm{~A}$ & $2-4 \mathrm{~mm}$ & $0.48 \pm 0.82$ & $0.91 \pm 1.58$ & $2.09 \pm 1.39$ & $2.65 \pm 2.3$ & $6.59 \pm 4.48$ & $5.72 \pm 1.81$ & $11.84 \pm 1.94$ & $6.74 \pm 5.5$ & $\begin{array}{c}11.83 \pm \\
1.16\end{array}$ & $8.89 \pm 2.29$ \\
\hline $\begin{array}{l}\text { Metalinhomoeus } \\
\text { numidicus }\end{array}$ & 2 & $e / f$ & $\mathrm{cr}$ & $1 \mathrm{~B}$ & $2-4 \mathrm{~mm}$ & $2.34 \pm 1.68$ & $5.58 \pm 1.35$ & $2.96 \pm 0.65$ & $6.99 \pm 2.72$ & $11.68 \pm 0.94$ & $5.72 \pm 1.81$ & $11.02 \pm 1.16$ & $14.42 \pm 2.9$ & $10.95 \pm 1.41$ & $10.54 \pm 1.6$ \\
\hline $\begin{array}{c}\text { Enoplolaimus } \\
\text { littoralis }\end{array}$ & 3 & $e / f$ & id & $2 \mathrm{~B}$ & $2-4 \mathrm{~mm}$ & $0.45 \pm 0.78$ & & $0.41 \pm 0.71$ & $0.43 \pm 0.74$ & & $0.83 \pm 1.07$ & $1.63 \pm 0.7$ & $1.91 \pm 2.18$ & $3.94 \pm 1.26$ & $1.62 \pm 0.68$ \\
\hline $\begin{array}{l}\text { Metoncholaimus } \\
\text { pristiurus }\end{array}$ & 3 & cla & pk & $2 \mathrm{~B}$ & $>4 \mathrm{~mm}$ & $8.31 \pm 1.43$ & $6.51 \pm 2.1$ & $8.03 \pm 1.74$ & $10.02 \pm 1.8$ & $10.76 \pm 1.13$ & $13.52 \pm 1.07$ & $8.17 \pm 3.08$ & $6.25 \pm 1.64$ & $6.15 \pm 2.05$ & $11.79 \pm 3.02$ \\
\hline $\begin{array}{l}\text { Odontophora } \\
\text { villoti }\end{array}$ & 2 & co & $\mathrm{cr}$ & $1 \mathrm{~B}$ & $2-4 \mathrm{~mm}$ & $0.45 \pm 0.78$ & & & $0.43 \pm 0.75$ & & & & $0.48 \pm 0.82$ & & \\
\hline $\begin{array}{l}\text { Oncholaimus } \\
\text { campylocer- } \\
\text { coides }\end{array}$ & 4 & cla & pk & $2 \mathrm{~B}$ & $>4 \mathrm{~mm}$ & $23.08 \pm 2.28$ & $15.84 \pm 3.08$ & $22.46 \pm 4.06$ & $24.44 \pm 1.5$ & $22.43 \pm 0.6$ & $25.82 \pm 1.27$ & $18.77 \pm 0.65$ & $23.56 \pm 2.26$ & $18 \pm 1.74$ & $23.18 \pm 1.3$ \\
\hline $\begin{array}{l}\text { Paracomesoma } \\
\text { dubium }\end{array}$ & 2 & cla & $\mathrm{sp}$ & $2 \mathrm{~A}$ & $1-2 \mathrm{~mm}$ & $0.45 \pm 0.78$ & $0.46 \pm 0.82$ & $0.41 \pm 0.71$ & $0.43 \pm 0.74$ & $0.92 \pm 0.8$ & $0.42 \pm 0.72$ & $0.81 \pm 1.41$ & $2.4 \pm 0.83$ & $1.75 \pm 0.76$ & $1.62 \pm 0.68$ \\
\hline $\begin{array}{c}\text { Paramonohystera } \\
\text { wieseri }\end{array}$ & 2 & cla & $\mathrm{cr}$ & 1B & $1-2 \mathrm{~mm}$ & $1.4 \pm 1.42$ & $2.32 \pm 1.6$ & $4.28 \pm 2.71$ & $6.99 \pm 2.72$ & $3.53 \pm 3.39$ & $3.28 \pm 0.73$ & $6.55 \pm 4.99$ & $3.36 \pm 1.66$ & $7.02 \pm 0.85$ & $1.2 \pm 2.09$ \\
\hline $\begin{array}{l}\text { Paramonohystera } \\
\text { pilosa }\end{array}$ & 2 & cla & $\mathrm{cr}$ & $1 \mathrm{~B}$ & $1-2 \mathrm{~mm}$ & $0.46 \pm 0.79$ & $0.46 \pm 0.8$ & $0.43 \pm 0.75$ & & & $1.23 \pm 1.22$ & $0.41 \pm 0.71$ & & $2.2 \pm 0.78$ & \\
\hline
\end{tabular}


Table 1. Cont.

\begin{tabular}{|c|c|c|c|c|c|c|c|c|c|c|c|c|c|c|c|}
\hline \multirow[t]{2}{*}{ Species } & \multicolumn{5}{|c|}{ Functional Traits } & \multicolumn{10}{|c|}{ Treatments } \\
\hline & $c-p$ & Tl & Am & FG & $\begin{array}{c}\text { Adult } \\
\text { Length }\end{array}$ & $\mathrm{CO}$ & $\mathbf{P}$ & S2 & S1 & A2 & A1 & SP2 & SP1 & AP2 & AP1 \\
\hline $\begin{array}{c}\text { Prochromadorella } \\
\text { longicaudata }\end{array}$ & 2 & $\mathrm{co}$ & id & $2 \mathrm{~A}$ & $1-2 \mathrm{~mm}$ & $42.38 \pm 0.9$ & $39.53 \pm 1.85$ & $22.06 \pm 2.33$ & $20.54 \pm 1.21$ & $19.82 \pm 2.96$ & $16.8 \pm 0.48$ & $13.47 \pm 1.22$ & $14.9 \pm 0.71$ & $7.01 \pm 1.45$ & $11.79 \pm 3.02$ \\
\hline $\begin{array}{l}\text { Spirinia } \\
\text { gerlachi }\end{array}$ & 3 & co & $\mathrm{cr}$ & $2 \mathrm{~A}$ & $1-2 \mathrm{~mm}$ & $0.46 \pm 0.79$ & & $1.7 \pm 0.75$ & $0.88 \pm 0.76$ & & $1.24 \pm 1.25$ & $0.41 \pm 0.71$ & $2.39 \pm 1.64$ & $0.43 \pm 0.75$ & $0.82 \pm 0.71$ \\
\hline Steineria sp. & 2 & cla & $\mathrm{cr}$ & $1 \mathrm{~B}$ & $1-2 \mathrm{~mm}$ & $0.46 \pm 0.79$ & & $0.41 \pm 0.71$ & & & & & $0.48 \pm 0.84$ & $1.31 \pm 1.32$ & \\
\hline $\begin{array}{c}\text { Synonchiella } \\
\text { edax }\end{array}$ & 4 & $e / f$ & $\mathrm{sp}$ & $2 \mathrm{~B}$ & $2-4 \mathrm{~mm}$ & $0.46 \pm 0.79$ & $0.93 \pm 0.81$ & $1.7 \pm 0.75$ & & & $0.82 \pm 0.71$ & $0.41 \pm 0.71$ & $1.93 \pm 0.84$ & $3.07 \pm 1.52$ & $1.65 \pm 1.43$ \\
\hline $\begin{array}{l}\text { Theristus } \\
\text { flevensis }\end{array}$ & 2 & $\mathrm{co}$ & $\mathrm{cr}$ & $1 \mathrm{~B}$ & $1-2 \mathrm{~mm}$ & $3.24 \pm 0.9$ & $5.13 \pm 2.2$ & $11.03 \pm 1.57$ & $4.83 \pm 2.1$ & $7.65 \pm 2.23$ & $2.04 \pm 0.69$ & $5.31 \pm 1.43$ & $5.29 \pm 1.69$ & $6.6 \pm 2.37$ & $4.06 \pm 0.62$ \\
\hline Viscosia cobbi & 3 & $e / f$ & $\mathrm{pk}$ & $2 \mathrm{~B}$ & $1-2 \mathrm{~mm}$ & $0.46 \pm 0.79$ & $0.48 \pm 0.82$ & $1.28 \pm 1.3$ & $3.5 \pm 0.79$ & $0.43 \pm 0.74$ & $0.42 \pm 0.7$ & & & & \\
\hline
\end{tabular}




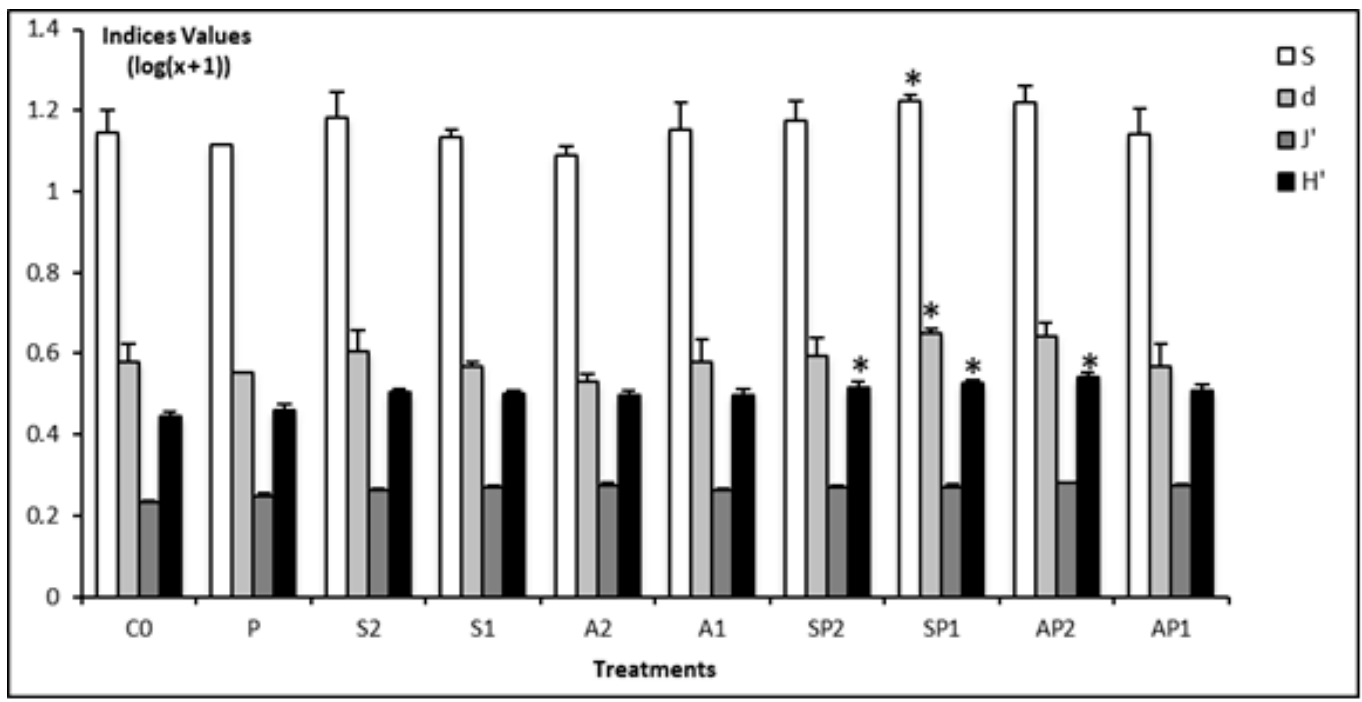

Figure 2. Graphical summary of univariate indices for nematode assemblages from each microcosm. $\mathrm{H}^{\prime}=$ Shannon-Wiener index, $\mathrm{d}=$ Margalef's species richness, $\mathrm{J}^{\prime}=$ Pielou's evenness, $\mathrm{S}=$ species number. An asterisk above bars indicates a significant difference $(p<0.05)$ with the control community $(\mathrm{C} 0) .\left(^{*}\right)$ above bars indicates significant difference $(p<0.05)$.

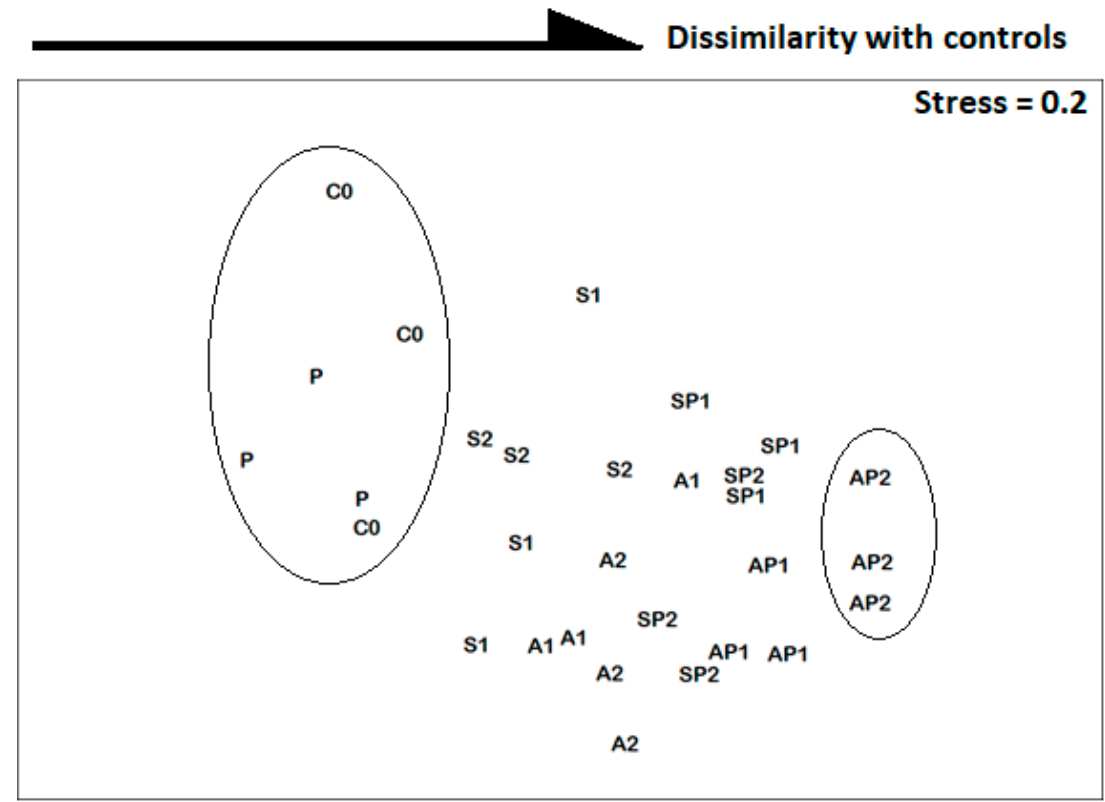

Figure 3. Non-metric multidimensional scaling (nMDS) 2D plot based on nematode species abundances from uncontaminated $(\mathrm{C} 0)$ and contaminated (P; S2; S1; A2; A1; SP2; SP1; AP2; AP1) microcosms.

The average dissimilarity value increased following the pattern " $\mathrm{P}$ " $\rightarrow$ "S1" $\rightarrow$ "S2" $\rightarrow$ "A2" $\rightarrow$ "A1" $\rightarrow$ "SP1" $\rightarrow$ "SP2" $\rightarrow$ "AP1" $\rightarrow$ "AP2" (Table 2). The ANOSIM results $(R$-statistics $=0.422-1, p$-value $<0.05$, Table 2$)$ indicated the presence of various taxonomic categories of nematodes, confirming the trends noticeable in the nMDS representation (Figure 3).

SIMPER results indicated that the abundance of $P$. longicaudata decreased in all treatments except for " $\mathrm{P}$ ", whereas the abundance of the nematode Marylynnia puncticaudata increased in the combined treatments, driving most of the noticed dissimilarity noticeable in the MDS graphic representation (Table 2). 


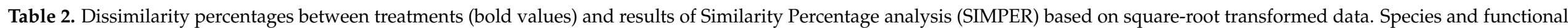
groups accounting for $\sim 70 \%$ of overall dissimilarity are ranked in order of importance of their contribution. More abundant (+); less abundant ( - ).

\begin{tabular}{|c|c|c|c|c|c|c|c|c|c|}
\hline & C0 vs. P & $\mathrm{C} 0$ vs. S2 & C0 vs. S1 & C0 vs. A2 & C0 vs. A1 & C0 vs. SP2 & C0 vs. SP1 & C0 vs. AP2 & C0 vs. AP1 \\
\hline $\begin{array}{c}\text { Average } \\
\text { dissimilarity (\%) }\end{array}$ & 20.04 & 29.04 & 28.67 & 33.11 & 34.17 & 38.66 & 35.35 & 47.55 & 39.79 \\
\hline$R$-statistics & 0.422 & 0.704 & 0.593 & 0.741 & 0.852 & 0.852 & 0.852 & 1 & 1 \\
\hline Species & $\begin{array}{c}\text { Oncholaimus } \\
\text { campylocercoides } \\
18.47 \%- \\
\text { Anticoma eberthi } \\
11.59 \%+ \\
\text { Metoncholaimus } \\
\text { pristiurus } 11.58 \% \\
- \\
\text { Prochromadorella } \\
\text { longicaudata } 8.8 \% \\
- \\
\text { Theristus flevensis } \\
8.45 \%+ \\
\text { Metalinhomoeus } \\
\text { numidicus } 8.02 \%+\end{array}$ & $\begin{array}{c}\text { Prochromadorella } \\
\text { longicaudata } \\
30.37 \%- \\
\text { Theristus flevensis } \\
14.44 \%+ \\
\\
\text { Anticoma eberthi } \\
10.65 \%+ \\
\text { Daptonema } \\
\text { trabeculosum } 6.84 \% \\
+ \\
\text { Oncholaimus } \\
\text { campylocercoides } \\
6.34 \%+\end{array}$ & $\begin{array}{c}\text { Prochromadorella } \\
\text { longicaudata } \\
35.16 \%- \\
\text { Paramonohysteria } \\
\text { weiseri } 10.15 \%+ \\
\\
\text { Metalinhomoeus } \\
\text { numidicus } 8.58 \%+ \\
\text { Viscosia cobbi } 5.48 \% \\
+ \\
\text { Anticoma eberthi } \\
5.48 \%+\end{array}$ & $\begin{array}{c}\text { Prochromadorella } \\
\text { longicaudata } \\
32.88 \%- \\
\text { Metalinhomoeus } \\
\text { numidicus } 14.4 \%+ \\
\text { Daptonema } \\
\text { trabeculosum } \\
10.34 \%+ \\
\text { Marylynnia } \\
\text { puncticaudata } \\
9.49 \%+\end{array}$ & $\begin{array}{c}\text { Prochromadorella } \\
\text { longicaudata } \\
32.36 \%- \\
\text { Anticoma eberthi } \\
11.44 \%+ \\
\\
\text { Metoncholaimus } \\
\text { pristiurus } 9.51 \%+ \\
\text { Oncholaimus } \\
\text { campylocercoides } \\
8.23 \%+ \\
\text { Marylynnia } \\
\text { puncticaudata } \\
8.23 \%+\end{array}$ & $\begin{array}{c}\text { Prochromadorella } \\
\text { longicaudata } \\
33.01 \%- \\
\text { Marylynnia } \\
\text { puncticaudata } \\
15.67 \%+ \\
\text { Metalinhomoeus } \\
\text { numidicus } 12.3 \%+\end{array}$ & $\begin{array}{c}\text { Prochromadorella } \\
\text { longicaudata } \\
40.58 \%- \\
\text { Metalinhomoeus } \\
\text { numidicus } 16.62 \% \\
+ \\
\text { Marylynnia } \\
\text { puncticaudata } \\
8.65 \%+ \\
\text { Daptonema } \\
\text { trabeculosum } 3.34 \% \\
+\end{array}$ & $\begin{array}{c}\text { Prochromadorella } \\
\text { longicaudata } \\
35.91 \%- \\
\text { Marylynnia } \\
\text { puncticaudata } \\
12.28 \%+ \\
\text { Metalinhomoeus } \\
\text { numidicus } 9.43 \%+ \\
\text { Daptonema } \\
\text { trabeculosum } 8.05 \% \\
+\end{array}$ & $\begin{array}{c}\text { Prochromadorella } \\
\text { longicaudata } \\
34.19 \%- \\
\text { Metalinhomoeus } \\
\text { numidicus } 11.36 \% \\
+ \\
\text { Marylynnia } \\
\text { puncticaudata } \\
11.35 \%+ \\
\text { Anticoma eberthi } \\
9.28 \%+\end{array}$ \\
\hline Feeding groups & $\begin{array}{c}12.36 \% \\
2 \mathrm{~B}- \\
1 \mathrm{~B}+\end{array}$ & $\begin{array}{l}23.49 \% \\
2 \mathrm{~A}-\end{array}$ & $\begin{array}{l}21.34 \% \\
2 \mathrm{~A}-\end{array}$ & $\begin{array}{c}27.36 \% \\
2 \mathrm{~A}-\end{array}$ & $\begin{array}{c}27.36 \% \\
2 \mathrm{~A}- \\
2 \mathrm{~B}+\end{array}$ & $\begin{array}{c}30.11 \% \\
2 \mathrm{~A}-\end{array}$ & $\begin{array}{c}25.22 \% \\
2 \mathrm{~A}-\end{array}$ & $\begin{array}{c}36.53 \% \\
2 \mathrm{~A}-\end{array}$ & $\begin{array}{c}31.82 \% \\
2 \mathrm{~A}-\end{array}$ \\
\hline Tail shape & $\begin{array}{l}9.09 \% \\
e / f+\end{array}$ & $\begin{array}{c}14.77 \% \\
\text { co - }\end{array}$ & $\begin{array}{c}20.16 \% \\
\text { co - }\end{array}$ & $\begin{array}{c}21.75 \% \\
\text { co - }\end{array}$ & $\begin{array}{c}30.58 \% \\
\text { co - }\end{array}$ & $\begin{array}{c}30.74 \% \\
\mathrm{e} / \mathrm{f}+\end{array}$ & $\begin{array}{c}23.93 \% \\
\text { co - }\end{array}$ & $\begin{array}{c}35.74 \% \\
\text { co - }\end{array}$ & $\begin{array}{c}35.22 \% \\
\mathrm{co}-\end{array}$ \\
\hline Amphid shape & $\begin{array}{c}8.50 \% \\
\mathrm{cr}+ \\
\mathrm{pk}-\end{array}$ & $\begin{array}{l}23.02 \% \\
\text { id - }\end{array}$ & $\begin{array}{l}23.78 \% \\
\text { id - }\end{array}$ & $\begin{array}{l}26.79 \% \\
\text { id - }\end{array}$ & $\begin{array}{l}29.29 \% \\
\text { id - }\end{array}$ & $\begin{array}{c}30.75 \% \\
\text { id - } \\
\text { cr }+\end{array}$ & $\begin{array}{l}31.62 \% \\
\text { id - }\end{array}$ & $\begin{array}{l}40.69 \% \\
\text { id - }\end{array}$ & $\begin{array}{c}33.91 \% \\
\text { id - } \\
\text { pk }+\end{array}$ \\
\hline Adult length & $\begin{array}{l}10.02 \% \\
>4 \mathrm{~mm}+\end{array}$ & $\begin{array}{c}9.02 \% \\
2-4 \mathrm{~mm}+\end{array}$ & $\begin{array}{c}12.09 \% \\
2-4 \mathrm{~mm}+\end{array}$ & $\begin{array}{c}13.74 \% \\
1-2 \mathrm{~mm}-\end{array}$ & $\begin{array}{c}23.64 \% \\
1-2 \mathrm{~mm}-\end{array}$ & $\begin{array}{c}26.69 \% \\
2-4 m m+\end{array}$ & $\begin{array}{c}23.76 \% \\
1-2 \mathrm{~mm}-\end{array}$ & $\begin{array}{c}25.52 \% \\
2-4 \mathrm{~mm}+\end{array}$ & $\begin{array}{c}30.91 \% \\
2-4 \mathrm{~mm}+\end{array}$ \\
\hline c-p score & $\begin{array}{l}11.86 \% \\
\text { cp2 }+\end{array}$ & $\begin{array}{l}7.13 \% \\
\text { cp2 }+\end{array}$ & $\begin{array}{l}9.58 \% \\
\text { cp3+ }\end{array}$ & $\begin{array}{l}7.52 \% \\
\text { cp3+ }\end{array}$ & $\begin{array}{c}13.25 \% \\
\text { cp3 }+\end{array}$ & $\begin{array}{l}9.96 \% \\
\text { cp3+ }\end{array}$ & $\begin{array}{l}11.53 \% \\
\mathrm{cp} 2-\end{array}$ & $\begin{array}{c}10.71 \% \\
\text { cp3 }+\end{array}$ & $\begin{array}{c}11.87 \% \\
\text { cp3+ }\end{array}$ \\
\hline
\end{tabular}




\subsection{Functional Diversity of Nematodes}

The C0 nematofauna was dominated by epigrowth feeders (2A) and omnivores carnivores (2B), with conical (co) and clavate (cla) tails, indistinct (id), pocket-like (pk) amphids, life history groups c-p 2, and length intervals $1-2 \mathrm{~mm}$. Except for treatment $\mathrm{P}$, the contamination led to a significant modification of feeding groups, amphid shape, tail shape, life history, and adult length composition, respectively (Figure 4).

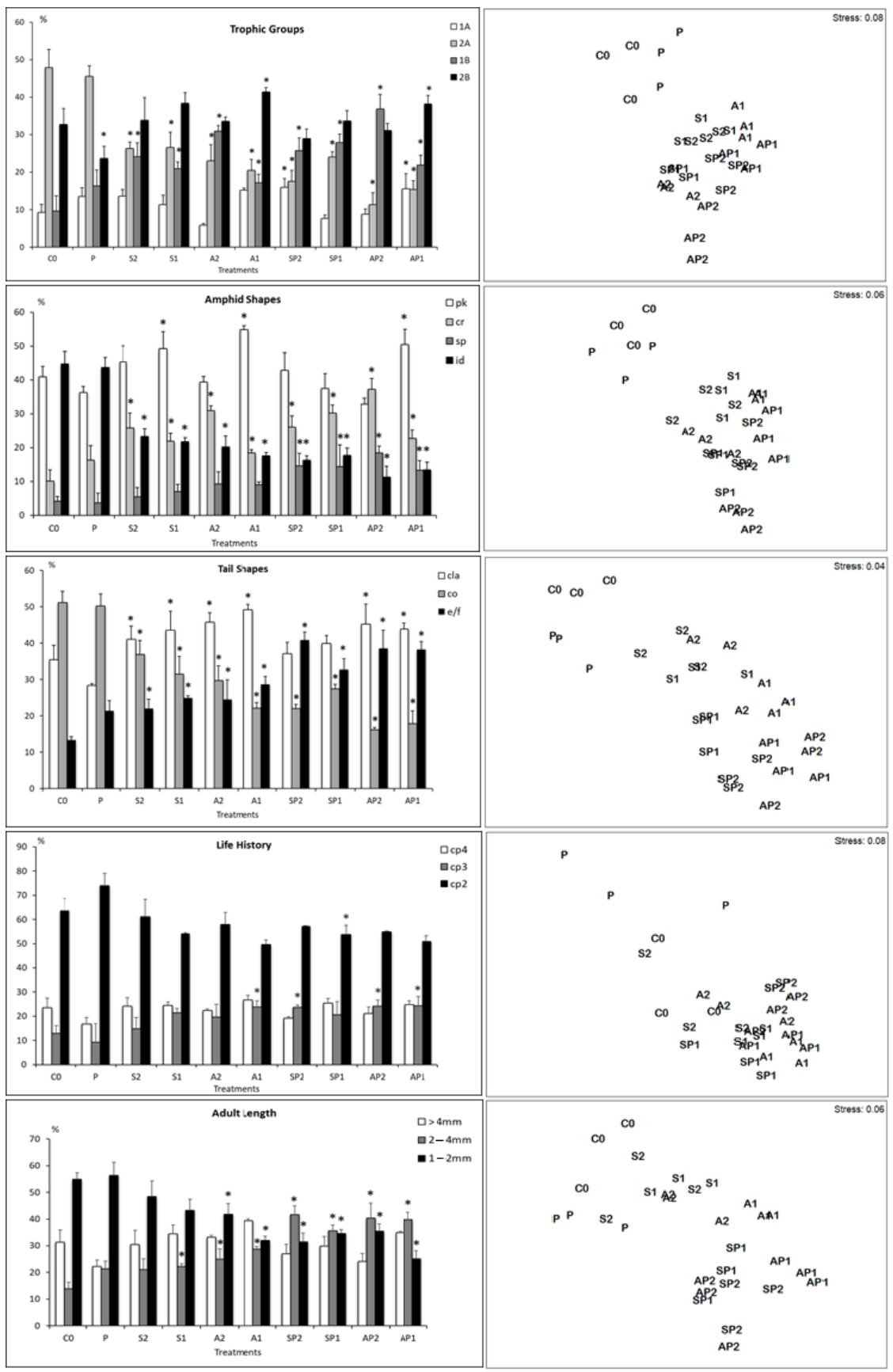

Figure 4. Non-metric multidimensional scaling (nMDS) 2D plots (right) and graphical summary (left) based on abundances of functional groups of nematode assemblages from each microcosm. Selective deposit feeders (1A); non-selective deposit feeders (1B); epigrowth feeders (2A); omnivorescarnivores (2B); short/round (s/r); elongated/filiform (e/f); conical (co); clavate/conical-cylindrical (cla); spiral (SP); pocket-like (Pk); indistinct (Id); circular (Cr). An asterisk above bars indicates a significant difference $(p<0.05)$ with control community $(\mathrm{C} 0)$. 
SIMPER results revealed a significant decrease in the abundance of the epigrowth guild (2A), with conical (co) tails, and indistinct (id) amphids compared to C0. Conversely, SIMPER results also revealed a significant increase in nematodes' abundance with a c-p score of 3, compared to $\mathrm{C} 0$. To a lesser extent, some treatments showed an increase in the number of taxa belonging to $1 \mathrm{~B}$ and $2 \mathrm{~B}$ guilds, with circular (cr) and pocket-like (pk) amphids, respectively (Table 2 ).

The nMDS second-stage ordination showed that the responses of nematode species to the different treatments depended mainly on their shapes of amphids $(86.45 \%)$, tails $(83.21 \%)$, and composition of trophic guilds (82.39\%) (Figure 5).

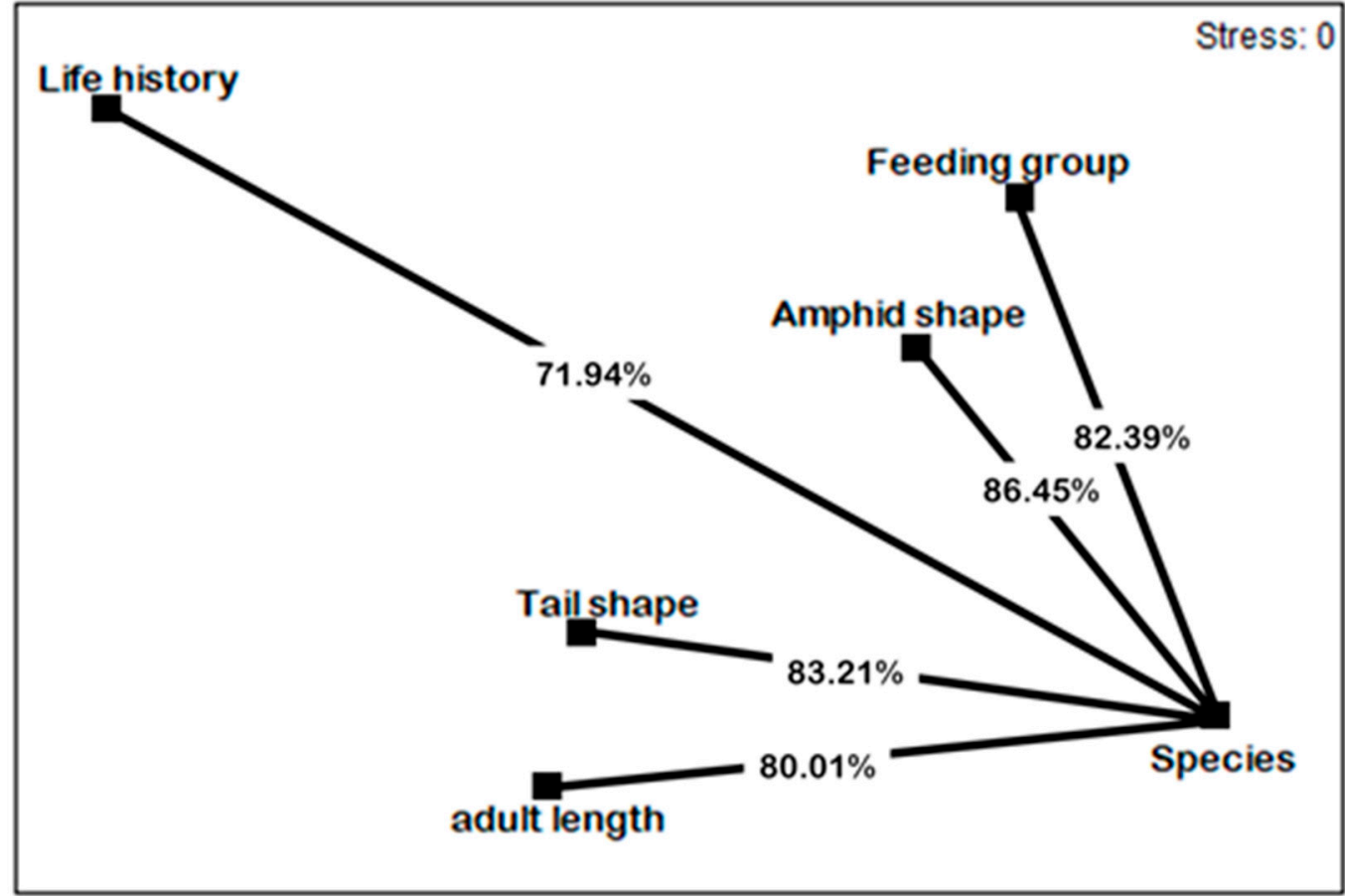

Figure 5. Non-metric multidimensional scaling (nMDS) second-stage ordination of inter-matrix rank correlations. For matrices included. See Figure 3 (species) and 4 (functional traits). Values indicate average similarity percentages between nMDS related to species and those related to functional traits.

\section{Discussion}

The control community was dominated by small-sized (1-2 mm), epigrowth feeding species (2A), dominated by P. longicaudata, along with larger ( $>4 \mathrm{~mm})$, omnivore-carnivore (2B) species, which was dominated by O. campylocercoides. The contamination with $\mathrm{P}$ showed no clear changes in the taxonomic composition of the nematode communities, except for the guild2B, which slightly decreased during the experiment. Species belonging to this trophic guild have large mouth openings and also ingest small grains as part of their diet [51], hence temptingly leading to a potential correlation between the level of contamination with microplastics and mortality rate of this group.

The knowledge of toxic effects of A on benthic invertebrates is scant. Previous investigations reported concentrations as low as $76 \mathrm{ng} . \mathrm{L}^{-1}$ in raw wastewater and 37 and $22.4 \mathrm{ng} . \mathrm{L}^{-1}$, respectively, in wastewater treatment plant outlets [52]. These concentrations of A imposed however little risk for benthic invertebrates [9]. The employed concentrations of A from the current experiment closely followed those previously reported for the marine amphipod Hyalella azteca LC50 [9]. Previous studies reported S in the inlet of Canadian wastewater treatment plants concentrations of $4 \mathrm{ng} . \mathrm{L}^{-1}$ and $1 \mathrm{ng} . \mathrm{L}^{-1}$, in the outlets, respectively. Previous experimental essays reported high toxic (LC50) effects of $S$ on harpacticoid copepods and larval grass shrimp for $96 \mathrm{~h}$ contaminations at $0.81 \mathrm{mg} . \mathrm{L}^{-1}$ and $1.18 \mathrm{mg} . \mathrm{L}^{-1}$, respectively [53]. The exposure to sublethal levels, reported by the same authors, showed several important consequences on the growth and development rate of harpacticoid 
copepods and assumed that $S$ might have had negative effects on growth-related traits. The current study showed a significant negative effect of $S$ on meiofauna abundance and on most measured functional traits of nematodes. The effects of $S$ contamination were comparable to that induced by A. These results also corroborate the comparable LC50s of these drugs observed on small amphipods.

Overall, the combined treatments AP and SP had stronger negative effects on the meiofauna community than each contaminant acting alone. These findings suggest that there was a synergic effect between polyvinyl chloride and these drugs. The increased surface to volume ratio of microplastics usually leads to high adsorption rates for various contaminants [23], such as heavy metals [22], antibiotics [24], and organic pollutants [21]. These chemicals bond with microplastics and penetrate living organisms following their ingestion, leading to toxicologic effects [54]. This could, at least in part, explain the significant decrease of meiofauna abundance in these treatments. However, according to the univariate indices, neither the A nor $S$ contamination alone, nor their combinations with $\mathrm{P}$, significantly influenced the nematode diversity. Still, the taxonomic composition of the nematode communities differed in treatments compared to control in terms of functional traits. Contrary to our expectations, at first glance it seems that the contamination actually proved to be beneficial for some taxa.

According to the SIMPER results (Table 2) M. puncticaudata was dominant in AP and SP treatments, but the abundance of $P$. longicaudata significantly decreased, in most treatments, except for P. Despite both species sharing several similarities it is possible that the differences in their body-size and life cycles to have led to the replacement of the latter group by the former. Likewise, the SIMPER results also indicated that Family Xyalidae (i.e., Theristus flevensis and Paramonohystera wieseri) mostly benefited from $\mathrm{S}$ treatment, same as Anticoma eberthi (in A1) and Metalinhomoeus numidicus (in A2) (Table 2).

The nMDS second stage (Figure 5) indicated that the shape of amphids' shape, followed by tail shape and feeding type, were the most important parameters affected in treatments. The amphid shape underwent a strong change with the decrease of indistinct shape (id) and the increase of circular (cr) and spiral (sp) shapes. Wakkaf et al. reported that the relative dominance of circular amphids' shape could explain their ability to detect and avoid pollutant.

\section{Conclusions}

Despite the wealth of ecotoxicological studies based on marine meiofauna as bioindicators, a considerable paucity of work related to the impact of microplastics on this diverse group is still present. To our knowledge, the current experiment is the first that investigated the effects of lipid regulators on meiofauna, with a main focus on free-living marine nematodes. Additionally, this study provided a better insight into the intimate interactions between atorvastatin, simvastatin, and microplastics, respectively.

The results of the current experiment revealed a significant toxic effect on meiofauna abundances. Interestingly, the Shannon index values for all treatments were close to control. However, SIMPER analysis reported significant modifications of nematodes diversity and identified the main functional traits that contributed most to the dissimilarity among control and treatments, namely that of epigrowth feeders (2A), with conical (co) tails and indistinct (id) amphids. Contamination with microplastics had noticeable impact only on the 2B trophic group, while the interactions between lipid regulator agents and microplastics revealed synergic interactions, resulting in increased toxicity of drugs.

Author Contributions: Conceptualization, M.A. (Mohamed Allouche), S.I. and F.B.; methodology, B.A. and M.A. (Mohamed Allouche); validation, A.H., M.B.A. and F.B.; formal analysis, B.A., M.A. (Mohamed Allouche), A.H. and M.B.A.; investigation, A.N. and F.B.; writing-original draft preparation, M.A. (Mohamed Allouche) and S.I.; writing—review and editing, M.A. (Mohammed Almalki), S.I., F.B. and O.P.; supervision, F.B.; project administration, B.A. and M.A. (Mohammed Almalki) All authors have read and agreed to the published version of the manuscript. 
Funding: This work was supported by the Tunisian Ministry of the High Education and Scientific Research. The authors are grateful to the Deanship of Scientific Research for funding this article by Taif University Research Supporting Project number (TURSP-2020/301), Taif University, Taif, Saudi Arabia. O.P. was supported by the National Core Program-Romanian Ministry of Research and Innovation Program, project 25 N 2019 BIODIVERS 19270103.

Institutional Review Board Statement: Not applicable.

Informed Consent Statement: Not applicable.

Data Availability Statement: The data are not shared due to restrictions, e.g., privacy and regulation.

Conflicts of Interest: The authors declare no conflict of interest.

\section{References}

1. Selamoglu, M. Importance of the cold chain logistics in the marketing process of aquatic products: An update study. J. Surv. Fish. Sci. 2021, 8, 25-29.

2. Cunningham, V.L.; Buzby, M.; Hutchinson, T.; Mastrocco, F.; Parke, N.; Roden, N. Effects of human pharmaceuticals on aquatic life: Next steps. Environ. Sci. Technol. 2006, 40, 3457-3462. [CrossRef]

3. Gore, M.; Sadosky, A.; Leslie, D.; Sheehan, A.H. Selecting an appropriate medication for treating neuropathic pain in patients with diabetes: A study using the UK and Germany Mediplus databases. Pain Pr. 2008, 8, 253-262. [CrossRef] [PubMed]

4. Grimmsmann, T.; Schwabe, U.; Himmel, W. The influence of hospitalisation on drug prescription in primary care-a large-scale follow-up study. Eur. J. Clin. Pharmacol. 2007, 63, 783-790. [CrossRef] [PubMed]

5. Ghoshdastidar, A.J.; Fox, S.; Tong, A.Z. The presence of the top prescribed pharmaceuticals in treated sewage effluents and receiving waters in Southwest Nova Scotia, Canada. Environ. Sci. Pollut. Res. 2015, 22, 689-700. [CrossRef] [PubMed]

6. Fuentes, A.; Pineda, M.; Venkata, K. Comprehension of top 200 prescribed drugs in the US as a resource for pharmacy teaching, training and practice. Pharmacy 2018, 6, 43. [CrossRef]

7. Mc Namara, K.; Alzubaidi, H.; Jackson, J.K. Cardiovascular disease as a leading cause of death: How are pharmacists getting involved? Integr. Pharm. Res. Pract. 2019, 8, 1-11. [CrossRef]

8. Wilson, S.; Simari, R.D.; Best, P.J.; Peterson, T.E.; Lerman, L.O.; Avirman, M.; Nath, K.A.; Holmes, D.R., Jr.; Lerman, A. Simvastatin preserves coronary endothelial function in hypercholesterolemia in the absence of lipid lowering. Arterioscler. Thromb. Vasc. Biol. 2001, 21, 122-128. [CrossRef]

9. Dussault, E.B.; Balakrishnan, V.K.; Sverko, E.D.; Solomon, K.R.; Sibley, P.K. Toxicity of human pharmaceuticals and personal care products to benthic invertebrates. Environ. Toxicol. Chem. 2008, 27, 425-432. [CrossRef]

10. Key, P.B.; Hoguet, J.; Reed, L.A.; Chung, K.W.; Fulton, M.H. Effects of the Statin Antihyperlipidemic Agent Simvastatin on Grass Shrimp, Palaemonetes pugio. Environ. Toxicol. 2008, 23, 153-160. [CrossRef]

11. Chen, X.Q.; Cho, S.J.; Li, Y.; Venkatesh, S. Prediction of Aqueous Solubility of Organic Compounds Using a Quantitative Structure-Property Relationship. J. Pharm. Sci. 2002, 91, 1838-1852. [CrossRef] [PubMed]

12. Estimation Program Interface (EPI) Suite. Available online: https://www2.epa.gov/tsca-screening-tools (accessed on 7 November 2020).

13. Strungaru, S.A.; Jijie, R.; Nicoara, M.; Plavan, G.; Faggio, C. Micro-(nano) plastics in freshwater ecosystems: Abundance, toxicological impact and quantification methodology. TrAC Trends Anal. Chem. 2019, 110, 116-128. [CrossRef]

14. Tagg, A.S.; Sapp, M.; Harrison, J.P.; Ojeda, J.J. Identification and quantification of microplastics in wastewater using focal plane array-based reflectance micro-FT-IR imaging. Anal. Chem. 2015, 87, 6032-6040. [CrossRef] [PubMed]

15. de Souza Machado, A.A.; Kloas, W.; Zarfl, C.; Hempel, S.; Rillig, M.C. Microplastics as an emerging threat to terrestrial ecosystems. Glob. Chang. Biol. 2018, 24, 1405-1416. [CrossRef] [PubMed]

16. Eerkes-Medrano, D.; Thompson, R.C.; Aldridge, D.C. Microplastics in freshwater systems: A review of the emerging threats, identification of knowledge gaps and prioritisation of research needs. Water. Res. 2015, 75, 63-82. [CrossRef]

17. Aytan, U.; Valente, A.; Senturk, Y.; Usta, R.; Esensoy Sahin, F.B.; Mazlum, R.E.; Agirbas, E. First evaluation of neustonic microplastics in Black Sea waters. Mar. Environ. Res. 2016, 119, 22-30. [CrossRef]

18. Andrady, A.L. The plastic in microplastics: A review. Mar. Pollut. Bull. 2017, 119, 12-22. [CrossRef]

19. Öztekin, A.; Bat, L. Microlitter Pollution in Sea Water: A Preliminary Study from Sinop Sarikum Coast of the Southern Black Sea. Turk. J. Fish. Aquat. Sci. 2017, 17, 1431-1440. [CrossRef]

20. Silva, A.B.; Bastos, A.S.; Justino, C.I.L.; da-Costa, J.P.; Duarte, A.C.; Rocha-Santos, T.A.P. Microplastics in the environment: Challenges in analytical chemistry-a review. Anal. Chim. Acta. 2018, 1017, 1-19. [CrossRef]

21. Heskett, M.; Takada, H.; Yamashita, R.; Yuyama, M.; Ito, M.; Geok, Y.B.; Ogata, Y.; Kwan, C.; Heckhausen, A.; Taylor, H.; et al. Measurement of persistent organic pollutants (POPs) in plastic resin pellets from remote islands: Toward establishment of background concentrations for international pellet watch. Mar. Pollut. Bull. 2012, 64, 445-448. [CrossRef] [PubMed]

22. Jasna, M.L.; Jelena, L.; Pero, T.; Varezić, D.B.; Šiljić, J.; Pribudić, J. Levels of trace metals on microplastic particles in beach sediments of the island of Vis, Adriatic Sea, Croatia. Mar. Pollut. Bull. 2018, 137, 231-236.

23. Hale, R.C.; Seeley, M.E.; La Guardia, M.J.; Mai, L.; Zeng, E.Y. A global perspective on microplastics. J. Geophys. Res. Ocean. 2020, 125. [CrossRef] 
24. Yu, Q.; Hu, X.; Yang, B.; Zhang, G.; Wang, J.; Ling, W. Distribution, abundance and risks of microplastics in the environment. Chemosphere 2020, 249, 126059. [CrossRef] [PubMed]

25. Warwick, R.M.; Price, R. Ecological and metabolic studies on free-living nematodes from an estuarine sand flat. Estuar. Coast. Mar. Sci. 1979, 9, 257-271. [CrossRef]

26. Guo, Y.; Somerfield, P.J.; Warwick, R.M.; Zhang, Z. Large-scale patterns in the community structure and biodiversity of free living nematodes in the Bohai Sea, China. J. Mar. Biol. Assoc. U.K. 2001, 81, 755-763. [CrossRef]

27. Balsamo, M.; Albertelli, G.; Ceccherelli, V.U.; Coccionia, R.; Colangeloc, M.A.; Curini-Gallettid, M.; Danovaroe, R.; D'Addabbof, R.; De Leonardisf, C.; Fabianob, M.; et al. Meiofauna of the Adriatic Sea: Present knowledge and future perspectives. Chem. Ecol. 2010, 26, 45-63. [CrossRef]

28. Moreno, M.; Semprucci, F.; Vezzulli, L.; Balsamo, M.; Fabiano, M.; Albertelli, G. The use of nematodes in assessing ecological quality status in the Mediterranean coastal ecosystems. Ecol. Indic. 2011, 11, 328-336. [CrossRef]

29. Semprucci, F.; Balsamo, M.; Appolloni, L.; Sandulli, R. Assessment of ecological quality status along the Apulian coasts (Eastern Mediterranean Sea) based on meiobenthic and nematode assemblages. Mar. Biodiver. 2018, 48, 105-115. [CrossRef]

30. Hedfi, A.; Ben Ali, M.; Nourel-Deen, A.; Albogami, B.; Hassan, M.; Saif, T.; Boufahja, F. Distribution and Abundance of Benthic Meiofauna in the Eastern Red Sea Coasts (Jeddah, Saudi Arabia). ARRB 2018, 26, 1-12. [CrossRef]

31. Mahmoudi, E.; Essid, N.; Beyrem, H.; Hedfi, A.; Boufahja, F.; Vtiello, P.; Aissa, P. Individual and combined effects of lead and zinc on a free-living marine nematode community: Results from microcosm experiments. J. Exp. Mar. Bio. Ecol. 2007, 343, 217-226. [CrossRef]

32. Hedfi, A.; Mahmoudi, E.; Boufahja, F.; Beyrem, H.; Aïssa, P. Effects of increasing levels of nickel contamination on structure of offshore nematode communities in experimental microcosms. Bull. Environ. Contam. Toxicol. 2007, 79, 345-349. [CrossRef]

33. Hedfi, A.; Boufahja, F.; Ben Ali, M.; Aïssa, P.; Mahmoudi, E.; Beyrem, H. Do trace metals (chromium, copper and nickel) influence toxicity of diesel fuel for free-living marine nematodes? Environ. Sci. Pollut. Res. 2013, 20, 3760-3770. [CrossRef]

34. Hedfi, A. Les Nématodes Libres, Outils Communautaires de Biosurveillance du Milieu Marin: Étude In Situ et en Microcosme. Ph.D. Thesis, Carthage University, Bizerte, Tunisia, 2008.

35. Schratzberger, M.; Whomersley, P.; Warr, K.; Bolam, S.G.; Rees, H.L. Colonisation of various types of sediment by estuarine nematodes via lateral infaunal migration: A laboratory study. Mar. Biol. 2004, 145, 69-78. [CrossRef]

36. Wakkaf, T.; Allouche, M.; Harrath, A.H.; Mansour, L.; Alwasel, S.; Ansari, K.G.M.T.; Beyrem, H.; Sellami, B.; Boufahja, F. The individual and combined effects of cadmium, polyvinyl chloride (PVC) microplastics and their polyalkylamines modified forms on meiobenthic features in a microcosm. Environ. Pollut. 2020, 266, 115263. [CrossRef]

37. Wieser, W. Benthic studies in buzzards bay. II. The meiofauna. Limnol. Oceanogr. 1960, 5, 121-137. [CrossRef]

38. Elarbaoui, S.; Richard, M.; Boufahja, F.; Mahmoudi, E.; Thomas-Guyonc, H. Effect of crude oil exposure and dispersant application on meiofauna: An intertidal mesocosm experiment. Environ. Sci. Process. Impacts. 2015, 17, 997-1004. [CrossRef] [PubMed]

39. Seinhorst, J.W. A rapid method for the transfer of nematodes from fixative to anhydrous glycerine. Nematologica. 1959, 4, 67-69. [CrossRef]

40. Platt, H.M.; Warwick, R.M.; Furstenberg, J.P. Free-living marine nematodes. Part, I. British Enoploids. S. Afr. J. Zool. 1985, 20, 177. [CrossRef]

41. Platt, H.M.; Warwick, R.M. Free-Living Marine Nematodes. Part II. British Chromadorids. Synopsis of the British Fauna (New Series No. 38); E.J. Brill/W.Backhuys: London, UK, 1988.

42. Warwick, R.M.; Platt, H.M.; Somerfield, P.J. Free-Living Marine Nematodes. Part III. British Monohysterids. Synopsis of British Fauna (New Series No. 53); Field Studies Council: London, UK, 1998.

43. Bezerra, T.N.; Decraemer, W.; Eisendle-Flockner, U.; Hodda, M.; Holovachov, O.; Leduc, D.; Miljutin, D.; Mokievsky, V.; Santiago, P.R.; Sharma, J.; et al. Nemys: World Database of Nematodes. 2020. Available online: http://nemys.ugent.be (accessed on 20 February 2021).

44. Wieser, W. Die Beziehung zwischen Mundhöhlengestalt, Ernäh rungsweiseund Vorkommen bei freilebenden marinen Nematoden. Arkiv. För. Zoologi. 1953, 2, 439-484.

45. Thistle, D.; Lambshead, P.J.D.; Sherman, K.M. Nematode tail-shape groups respond to environmental differences in the deep-sea. Vie Milieu. 1995, 45, 107-115.

46. Bongers, T.; de Goede, R.G.M.; Korthals, G.W.; Yeates, G.W. An update to the cprating of nematode genera can be found in proposed changes of c-p classification for nematodes. Russ. J. Nematol. 1995, 3, 61-62.

47. Schratzberger, M.; Warr, K.; Rogers, S.I. Functional diversity of nematode communities in the southwestern North Sea. Mar. Env. Res. 2007, 63, 368-389. [CrossRef]

48. Clarke, K.R. Non-parametric multivariate analyses of changes in community structure. Aust. J. Ecol. 1993, 18, 117-143. [CrossRef]

49. Clarke, K.R.; Warwick, R.M. Change in Marine Communities: An Approach to Statistical Analysis and Interpretation, 2nd ed.; PRIMER-E., Ltd., Plymouth Marine Laboratory: London, UK, 2001.

50. Clarke, K.R.; Gorley, R.N. PRIMER v5: User Manual/Tutorial; PRIMER-E: London, UK, 2001.

51. Allouche, M.; Nasri, A.; Harrath, A.H.; Mansour, L.; Alwasel, S.; Beyrem, H.; Plăvan, G.; Rohal-Lupher, M.; Boufahja, F. Meiobenthic nematode Oncholaimus campylocercoides as a model in laboratory studies: Selection, culture, and fluorescence microscopy After exposure to phenanthrene and chrysene. Environ. Sci. Pollut. Res. 2021, 28, 21303-21313. [CrossRef] [PubMed] 
52. Miao, X.-S.; Metcalfe, C.D. Determination of cholesterol-lowering statin drugs in aqueous samples using liquid chromatographyelectrospray ionization tandem mass spectrometry. J. Chromatogr. A 2003, 998, 133-141. [CrossRef]

53. Dahl, U.; Gorokhova, E.; Breitholtz, M. Application of growth-related sublethal endpoints in ecotoxicological assessments using a harpacticoid copepod. Aquat. Toxicol. 2006, 77, 433-438. [CrossRef] [PubMed]

54. Kirstein, I.; Kirmizi, S.; Wichels, A.; Garin-Fernandez, A.; Erler, R.; Löder, M.; Gerdts, G. Dangerous hitchhikers? Evidence for potentially pathogenic Vibrio spp. on microplastic particles. Mar. Environ. Res. 2016, 120, 1-8. [CrossRef] 